\title{
Keywords, Volume 8, 2003
}

\begin{tabular}{|c|c|c|c|c|c|}
\hline Addiction & 266 & Financial decisions & 82 & Morale & 178 \\
\hline Admission & 48 & France & 3 & Multidimensional & 252 \\
\hline Age-related visual impairmen & 168 & Geopolitics & 238 & Neuroticism & 279 \\
\hline 131, 160, 178, & 192,200 & German-Anglo-American & & Odor memory & 77 \\
\hline Alzheimer's disease & 148 & comparison & 87 & Personality & 00,223 \\
\hline Anxiety & 252 & Germany & 18 & $\mathrm{PhD}$ & \\
\hline Apprenticeship & 34 & Gerontology & 200 & Political map & 23 \\
\hline Attentional bias & 252 & Gerontopsychology & 87 & Psychological control & 168 \\
\hline Attitudes toward currencies & 82 & Graduate school & 9 & Psychology & \\
\hline Autobiographical memory & 192 & Graduate student & 48 & Psychosemantics & \\
\hline Behavior change strategies & 66 & Graduate training & 18 & Psychosocial adaptation & 16 \\
\hline Bibliometrical analysis & 87 & Happiness & 178 & Risk perception & 26 \\
\hline Big Five & 131 & Higher education & 23,48 & Self-efficacy & 13 \\
\hline Brain plasticity & 148 & History of psychology & 87 & Self-mechanisms & 20 \\
\hline Cognitive plasticity & 148 & Human development & 101 & Self-regulation & 27 \\
\hline Consciousness & 77 & Human values & 101 & Semantic space & 23 \\
\hline Denial & 266 & Individual differences & 279 & Social network & 16 \\
\hline Developmental psychology & 87 & Individualism & 101 & Social-emotional characteristics & 22 \\
\hline Dissertation & 9 & Information search & 82 & Standardization "learning-alliance & $\mathrm{ce}^{\prime \prime}$ \\
\hline Dissonance theory & 82 & Intelligence & 200 & Stereotypes & 23 \\
\hline Diversity & 200 & Interpretative bias & 252 & Strategies and interferences & \\
\hline Doctoral degree & $23,28,48$ & Italy & 23 & Student supervision & 28,48 \\
\hline Doctoral education & 34 & Learning potential & 148 & Subjective well-being & 17 \\
\hline Doctoral programs & 18 & Legislation & 28 & Supervisor & \\
\hline Doctoral studies & 28 & Life events & 192 & Sustainable development & 6 \\
\hline Doctoral training & 3,9 & Life satisfaction & 160,178 & Testing-the-limits & 148 \\
\hline Dynamic assessment & 148 & Lifespan psychology & 192 & Urban/rural & 16 \\
\hline Dynamics & 192 & Marital instability & 223 & Values & 131 \\
\hline Environmental psychology & 66 & Marital quality & 223 & Well-being & 13 \\
\hline Euro & 82 & Memory bias & 252 & & \\
\hline Extraversion & 279 & Mild cognitive impairment & 148 & & \\
\hline
\end{tabular}

